\title{
Deciphering the transcriptomic response of Ilyonectria robusta in relation to Ginsenoside Rg1 treatment and the development of panax ginseng rusted root disease
}

\section{Qiong Li}

Jilin Agricultural University https://orcid.org/0000-0003-3322-9365

\section{Yonghua Xu}

Jilin Agricultural University

\section{Lianxue Zhang}

Jilin Agricultural University

\section{Peng Di}

Jilin Agricultural University

\section{Baohui Lu}

Jilin Agricultural University

Changbao Chen ( $\nabla$ ccb2021@126.com)

https://orcid.org/0000-0001-8448-8098

\section{Research article}

Keywords: Panax ginseng, RNA-Seq, ginsenoside, llyonectria robusta

Posted Date: November 28th, 2019

DOI: https://doi.org/10.21203/rs.2.17833/v1

License: (c) (i) This work is licensed under a Creative Commons Attribution 4.0 International License.

Read Full License 


\section{Abstract}

Rust rot is a severe Panax ginseng disease caused by llyonectria robusta. The severity of the disease is related to the content of residual ginsenoside content in the soil. Herein, the effects of different concentrations of ginsenoside Rg1 on ginseng rust rot and its pathogens were studied, and the growth and sporulation of fungi cultured in vitro were determined. The results showed that Rg1 significantly promoted the mycelial growth, and sporulation compared to the control, and aggravated the disease symptoms of Panax ginseng. A total of 6,708 transcripts out of 213,131 annotated genes identified from global transcriptomic analysis were differentially expressed in I.robusta grown in the Rg1 treatment. These genes were found to be related to the carbon-nitrogen metabolism, transport, and assimilation. Many of these genes were also associated with pathogenicity based on the Phi-base database. Several transcription factors were related to specific biological processes, such as nitrogen utilization. The current results revelation that Rg1 plays a major role in the development of rust rot by promoting fungal cell growth and affecting the expression of genes required for pathogenesis. Rg1 could aggravate the invasion of I.robusta on ginseng root, which preliminarily revealed the reason for the aggravation of rust rot in ginseng $\mathrm{c}$ ontinuous cropping obstacle.

\section{Background}

Ginseng (Panax ginseng C.A.Mey.) is a highly valuable herbaceous perennial plant (Araliaceae family). Ginseng cultivation has made progress since last century. However, continuous cropping obstacle is a major threat to the growth and sustainable production of ginseng and is mainly caused by autotoxicity and pathogen accumulation [1]. Previous studies have shown that ginseng metabolites have autotoxic effects[2].however, the aggravation of the disease caused by continuous cropping obstacle is rarely reported. Nicol et al. isolated ginsenosides $\mathrm{F} 11, \mathrm{Rb} 1, \mathrm{Rb} 2, \mathrm{Rc}, \mathrm{Rd}, \mathrm{Re}$, and $\mathrm{Rg} 1$ from the rhizosphere soil of American ginseng [3]. These ginsenosides could be used as allelochemicals for rhizosphere fungi growth, which might be one of the factors of continuous cropping obstacle[4]. Furthermore, saponins are also speculated to act as allelopathic mediators of plant-fungal interactions. It has been shown that total ginsenoside part (T-GF), propanaxadiol type ginsenoside part (PPD-GF), and propanaxatriol type ginsenoside part (PPT-GF) promoted the growth of pathogen [5].

Interestingly, a variety of physical and chemical factors in the soil combined with the plant compounds affect the growth of soil microorganisms, but the exact mechanism is unclear [6]. Fungi can respond to changes in the quantity and quality of compounds through complex regulatory mechanisms[4]. Rust rot fungus of ginseng is one of the diseases that hinders the ginseng continuous cropping obstacle, it is an important problem to be solved in ginseng planting [7]. Thus, we studied the development of ginseng rust rot under the treatment of different concentration of Rg1.we also analyzed and compared the transcriptomes of Ilyonectria robusta under Rg1 treatment to study the response mechanisms and identify the major genes and biological processes in I. robusta affected by Rg1 treatment. The present study reported the first transcriptome profile of I. robusta and its response to Rg1 treatment. we also identified candidate genes involved in the continuous cropping obstacle of P. ginseng. 


\section{Results}

Disease severity of P. ginseng in response to different concentrations of ginsenoside $\mathrm{Rg} 1$

Rust spot (Fig. 1A) was observed in control plants (without Rg1; Control) and plants treated with different concentrations of $\operatorname{Rg} 1(0.5,1$, and $2 \mathrm{mM})$ during DAl 30 treatment. Compared to the control, the disease severity index of ginseng rust increased in a Rg1 concentration-dependent manner (Fig. 1B). When the concentration of Rg1 was $0.5 \mathrm{mM}$, the disease began to worsen, which increased by $32.43 \%$ as compared to the control. When the concentration of $\mathrm{Rg} 1$ increased to 1 and $2 \mathrm{mM}$, the disease severity index increased by $80.38 \%$ and $87.79 \%$, respectively.

Figure 1. The disease symptom (A) and disease severity index $(B)$ of ginseng plants treated with different concentrations of ginsenoside Rg1: $0.5 \mathrm{mM}$ (A2), $1 \mathrm{mM}$ (A3), $2 \mathrm{mM}$ (A4), and no Rg1 control (A1). (A) Images acquired on day 30 after inoculation. The average severity index of the cylindrical root rot of $(B)$ ginseng (\%).

Characterization of I. robusta cultured in different concentrations of ginsenoside Rg1

The characteristics of I. robusta cultured with different concentrations of ginsenoside Rg1 to observe the phenotypic changes of I. robusta. Compared to Control, the density, diameter, and color of the colony increased with the increase in concentration of ginsenoside Rg1 (Fig. 2D). Also, I. robusta grown in different treatment concentration of Rg1 showed significant differences in the sporulation yield (Fig. 2A), cell density under logistic increment (Fig. 2B), and The mycelium growth of I. robusta growing in Rg1 increased with increase Rg1 concentration (Fig. 2C). The results showed that the growth of I. robusta was enhanced in $\mathrm{Rg} 1$ treatment. These observations point to a mechanism by which $\mathrm{Rg} 1$ concentrations in the soil increase to a certain level to cause more serious ginseng rust rot disease.

Figure 2. Effects of ginsenoside Rg1 on the growth of I. robusta cultured in vitro. (A) Sporulation production at DAI 3 (3 days after inoculation); (B) Fungal cell densities (OD600) at logistic increment; (C) Fungal mycelial growth at increment; (D) Colonies of I. robusta observed at DAI 3 in no ginsenoside Rg1 control (D1, top view; D2, bottom view), 0.5 mM ginsenoside Rg1 (D3, top view; D4, bottom view), $1 \mathrm{mM}$ ginsenoside Rg1 (D5, top view; D6, bottom view), and 2mM ginsenoside Rg1 (D7, top view; D8, bottom view). The different letters in Fig. $2 A$ and Fig. $2 B$ indicate a highly significant difference $(P<0.01)$. The error bar represents the standard deviation of at least three repeats.

Illumina sequencing, de novo assembly, and gene annotation

The transcripts of Illumina sequencing, ab initio assembly and gene annotation, and Rg1 treatment of independent biological samples were sequenced to produce a total of 352959960 original RNA sequences. (Table 1). All original sequence reads are saved in the NCBI sequence read file (BioProject ID: PRJNA575915). In addition, 26006870987 and 25343652159, Q20\% (base mass $>20 \%$ ) clean-paired end 
sequence reads were produced in the control group and $\mathrm{Rg} 1$ treatment group, respectively (Table 2). Based on the GO horizontal annotation, 32,625 single genes were divided into three categories: cellular component (cc), molecular functional (mf), and biological process (bp) (Fig. 3A). In the KEGG annotation analysis, 48,256 unigenes were identified and divided into seven metabolic pathways (metabolism, genetic information processing, environmental information processing, cellular processes, organismal systems, human diseases, and drug development).

Figure 3.Transcriptome functional annotation analysis. (A) GO classification of the unigenes. Based on the functional annotation, these unigenes were grouped into three categories: biological process, cellular component, and molecular function. (B) KEGG classified these metabolic pathways into seven categories: metabolism, genetic information processing, environmental information processing, cellular processes, organismal systems, human diseases, drug development).

Table 1. Summary of I. robusta mycelium transcriptome data obtained in this study

\begin{tabular}{lllllll} 
Sample & Raw reads & Raw bases & Clean reads & Clean bases & Q20 (\%) & Q30 (\%) \\
\hline CK_Third1 & 49702570 & 7505088070 & 48391680 & 7207952662 & 98.79 & 96.2 \\
\hline CK_Third2 & 65590538 & 9904171238 & 64156582 & 9556773169 & 98.88 & 96.43 \\
\hline CK_Third3 & 63762930 & 9628202430 & 62116156 & 9242145156 & 98.89 & 96.47 \\
\hline total & 179056038 & 27037461738 & 174664418 & 26006870987 & & \\
\hline G_Third1 & 52424732 & 7916134532 & 51073008 & 7614818082 & 98.88 & 96.41 \\
\hline G_Third2 & 63197160 & 9542771160 & 61819738 & 9217635830 & 98.88 & 96.41 \\
\hline G_Third3 & 58282030 & 8800586530 & 57040238 & 8511198247 & 98.9 & 96.48 \\
\hline total & 173903922 & 26259492222 & 169932984 & 25343652159 & &
\end{tabular}

Elucidation of biological functions altered in response to $\mathrm{Rg} 1$ treatment

Analyze the results. Among 213131 unigenes, 7735 showed differential expression between the two groups (Fig. 2). In order to study the changes in the I. robusta transcriptional group caused by Rg1 treatment, a GO/KEGG enrichment analysis was performed in the control and Rg1 treatment groups $(p<0.05)$. Taken together, KEGG terminology annotation analysis of $\mathrm{Rg} 1$ induced the expression of related genes in multiple pathways, with pyruvate (KO00620), propionate (KO00640), alanine, aspartic acid, and glutamic acid (KO00250) at the top of the pathway enrichment analysis (Table S1) related to carbohydrate or amino acid metabolism[8]. It is speculated that ammonia can be used as a nitrogen source of fungi, which maximizes the growth of the total population, and the digestibility of carbohydrates is the main factor to control the growth and reproduction of fungi[9]. 
Figure 4. Differential gene enrichment analysis. (A) GO enrichment analysis and (B) KEGG enrichment analysis. The ratio of the number of unigenes commented to the GO/KEGG term in the gene set to the number of unigenes commented to the GO/KEGG term. The larger the ratio, the greater the degree of enrichment. The size of the point represents the number of unigenes in the GO/KEGG term, and the color of the point corresponds to a different FDR (pvaule_corrected) range.

Rg1 treatment affects the pathogen-host interaction.

In order to determine the potential virulence and pathogenicity-related genes of destructive I. robusta, the function of the whole single gene in the pathogen-host interaction (Phi) gene database (http://www.phibase.org) was analyzed by BLAST (cutoff E value $\leq 10^{-5}$ ). This database is a collection of genes that indicate the effect of the results of pathogen-host interactions from fungi, oomycetes, and bacteria[10]. After selecting the DEGs with log2 multiplier $\geq 2$ in different Rg1 treatments, 26 hypothetical pathogenhost interaction related genes were identified (Table S2). A number of Fusarium orthologs were found in the cereal pathogenic fungi I. robusta In Fusarium graminearum (3 genes), Fusarium oxysporum (2 genes), pseudoBurkholderia (2 genes), and Candida albicans (2 genes), a large number of Fusarium protoplasts were found in Campylobacter jejuni ( 2 genes) and Mycobacterium tuberculosis (3 genes). Salmonella enteritis (2 genes) and others (9 genes)[11]. This analysis revealed CK vs Rg1 DEGs in the Phi gene (Fig. 6A). Phi database demonstrated that 13 (50\%) genes did not have any effect on the fungal pathogenicity and they were considered to be necessary for the cell communication/signal transduction, metabolism, and transcription between pathogen and host[8]. Other genes were associated with decreased virulence (10 genes; $38.5 \%$ ), loss of virulence ( 2 genes; $7.7 \%$ ), and increased virulence (1 gene; 3.8\%) (Fig. 5A).

Among the 26 genes, 10 were upregulated and 16 were downregulated after Rg1 treatment, of which, the increased_virulence gene was upregulated (Fig. 5B). The overall changes in the expression of Phi-related genes showed that their expression was greatly affected by Rg1. This phenomenon explicated the effect of $\mathrm{Rg} 1$ on the severity of destructive rust rot disease in $P$. ginseng.

Figure 5. (A) DEGs related to Phi and the phenotype of pathogen. (B)Bar graph presents the expression estimates for genes differentially regulated according to the Rg1 treatment. 
Figure 6. (A) Cluster analysis of gene expression patterns selected in Phi. (B) Cluster analysis of cell growth-related gene expression patterns.

Figure 7. qRT-PCR analysis of selected genes.

Cell growth is affected by $\mathrm{Rg} 1$ treatment

All nitrogen uptake and transformation processes are mediated by enzyme systems, which require carbon, nitrogen, and energy for synthesis and expression. The current analysis identified the differential expression of carbon, nitrogen, and energy-related genes (Table S2). In addition, the up- or downregulation responses after Rg1 treatment differed significantly (Fig. 6B). Cluster analysis of cell growth-related gene expression patterns revealed that the expression of genes for nicotinamide adenine dinucleotide phosphate (NADP) or nicotinamide adenine dinucleotide (NAD)-dependent glutamate dehydrogenase (NADP/NAD-GDH) was highly variable (FDR-corrected P-value $<0.01$ ) when the pathogen was treated in Rg1. The expression of these genes was also validated by qRT-PCR (Fig. 7). These results suggested that glutamate plays a key role in cell growth, which is consistent with the results of previous studies[9].

The expression of aminomethyltransferase was significantly increased in Rg1 treatment, which possible promoted the release of ammonia (ammonia) and the transfer of methylene carbon unit to tetrahydrofolate moiety (4.06-fold change in Rg1 versus control). Consecutively, the expression of carbonnitrogen ligase, transaminase, and L-glutamate transmembrane transporter showed 3.75, 33.78, and 2.23fold higher than that of the control after Rg1 treatment, respectively. The results also showed significant differences in the expression of transporters $\mathbb{( F D R}$-corrected $\mathrm{P}<0.01$ ). These results of great significance for the effective utilization of carbon and nitrogen in Rg1-treatements cells.

\section{Discussion}

Ginsenoside Rg1 can change the infectivity of pathogenic bacteria[12]; however, the molecular mechanism of Rg1 affecting the results of plant pathogen interaction is not clear. The major factors may include regulation of pathogenic gene expression, and influence interaction between the host and the pathogen. Host-pathogen interactions are complex, including (i) the ability to recognize compatible hosts/pathogens; (ii) the ability of hosts to either tolerate the presence of a pathogen or a plant's susceptibility to pathogens; and (iii) growth or spore production of fungi and the ability of pathogens to colonize host cells[13]. The results presented here showed that Rg1 promotes the mycelial growth, cell density, pore production, and the disease development of P. ginseng.

Fungi have developed different mechanisms for absorbing and assimilating minerals and organic forms of nitrogen, enabling the usage of a wide range of organic and mineral compounds[14, 15]. Thus, we 
performed global transcriptome analysis through RNA sequencing to enhance our understanding of the response mechanisms of I. robusta to Rg1 treatment. The results of KEGG/GO enrichment $(P<0.0001)$ showed that Rg1 could regulate the biosynthesis of general proteins, the transport of carbon and nitrogen compounds, and the transmembrane transport of amino acids in I. robusta, and I. robusta treated with Rg1 altered the expression of key genes in the carbon-nitrogen uptake pathway. Also, the expression of genes encoding high affinity permeation was increased to maximize the absorption of limited carbonnitrogen sources[16, 17].

A total of 26 genes (Table S3) were putatively associated with host plant-pathogen interactions in I. robusta cultured under Rg1. Of these genes, several were associated with reduced virulence $(10 ; 38.5 \%)$, loss of pathogenicity (3;7.7\%), and increased virulence (1;3.8\%) (Fig. 3A). Included among this set of 26 genes were orthologs of Cfmc Boaa, Vtlr, Vatr1, Famyo2, Mosfa1, Groel, Eprs, Fsr1, Tsr, Thioredoxin 1 and Cspv. For example, the expression of Groel induced by Rg1 increased about 3.459-fold, which is critical for improving the virulence and high pathogenicity of Porphyromonas gingivalis[18]. Under the induction of Rg1, the expression of Mosfa1 increased 3.25-fold, which was related to the growth of infectious mycelium and the infection rate of Magnaporthe grisea[19]. Rg1 upregulates the expression of Fsr1 and plays a critical role in iron metabolism, which is related to the pathogenicity and virulence of Fusarium verticillioides[20]. However, 13 genes (50\%) were not necessary for pathogenicity, but were previously found to be associated with Phi. Gnn1 is a types of glycogen, which is involved in the pathogenicity of Fusarium oxysporum to plant hosts[21]. The striatal homologue fsr1 forms an intimal-related complex that regulates the virulence of Fusarium oxysporum, a maize pathogen. The MAP1 gene of Fusarium graminearum is the key gene for the pathogenesis and development of peritonitis[22]. These results showed that Rg1 treatment affects the results of interaction with ginseng through a variety of mechanisms, including the changes in pathogenicity.

\section{Conclusions}

The correlation between Rg1 and I. robusta is complex. Studies presented here emphasized the role of Rg1 as important aspects in I. robusta pathogenesis. The number of DEGs associated with Phi increased by $57 \%$, indicating that $\mathrm{l}$. robusta has a strong response to $\mathrm{Rg} 1$ through plant-pathogen interaction genes and Multiple related pathways of gene regulation affect I. robusta. These results supported the hypothesis that Rg1 could aggravate the invasion of llyonectria robusta on ginseng root, which preliminarily revealed the reason for the aggravation of rust and rot in ginseng continuous cropping process.

\section{Methods}

\subsection{Assays of I. robusta disease}

The physiologically mature seeds of P. ginseng were soaked for 20 min with $150 \mathrm{ppm}$ carbendazim. Then, twenty-five seeds were planted in a $2 \mathrm{~L}$ tank in April 2017 and filled with a mixture of vermiculite 
and ash (inert crushed volcanic rock) (1:1 v/v). The seedlings were rinsed with sterile water for 30 days, followed by microinjection of $100 \mu \mathrm{L}(107 \mathrm{CFU} / \mathrm{mL})$ spore suspension of I. robusta using an aseptic syringe. Simultaneously, different concentrations of Rg1 (sterile water, 0.50, 1.00, and $2.00 \mathrm{mM}$ ) were added to each basin, and each treatment was repeated three times. The inoculated plants were grown in an insect-proof greenhouse at $28^{\circ} \mathrm{C}$ with a photoperiod of $16 \mathrm{~h}$ light/ $8 \mathrm{~h}$ dark[23]. A randomized complete block design with three replicates was carried out on the inoculated $P$. ginseng plants fertilized using different concentrations of Rg1. No Rg1 treatment was used as the control.

Each plot was evaluated independently according to the symptoms of all roots. The symptom severity score, 0 (asymptomatic) to 4, was used. Grade was mild rust spot in the root epidermis, grade 2 was large area rust spot in the root epidermis, grade 3 was local necrosis caused by rust fungus, and grade 4 was large area rot and death of root. Disease severity index (DSI) of each replicate was calculated as DSI = $100 \times(\Sigma$ score $/ 4 \times N) ; N=$ total number of plants $(N=25)$. SAS9.4 software (SAS Institute Inc.) was used for two-way analysis of variance.

1.

2.2. Culturing of I. robusta

I. robusta is a ginseng pathogen isolated from Jilin Province, China in The isolates were stored on potato glucose agar slant at $4{ }^{\circ} \mathrm{C}$ until The conidia were cultured in improved Czapek medium (containing per L: $1 \mathrm{~g} \mathrm{~K} 2 \mathrm{HPO} 4,0.5 \mathrm{~g} \mathrm{MgSO} 4 \cdot 7 \mathrm{H} 2 \mathrm{O}, 0.5 \mathrm{~g} \mathrm{KCl}, 0.01 \mathrm{~g} \mathrm{Fe} 2 \mathrm{SO} 4,30 \mathrm{~g}$ sucrose and Rg1 at the same total concentration of $0.50 \mathrm{mM}, 1.00 \mathrm{mM}$ or $2.00 \mathrm{mM}$ ). The mixture was agitated at $250 \mathrm{rpm}$ and $25^{\circ} \mathrm{C}$ on a rotating vibrating screen for 3 days[24]. The fungal growth was measured by optical density at $600 \mathrm{~nm}$ (OD600) on a 21 D spectrophotometer (Milton Roy, Chicago, IL, USA). Mycelium growth was measured on agar plates. Three biological replicates of destructive I. robusta mycelium grown in $\operatorname{Rg} 1(1 \mathrm{Mm}$ treatment)were assessed. Total RNA isolation and RNA-seq analysis. Total RNA of the mycelium was extracted and obtained by centrifugation at $4^{\circ} \mathrm{C}$ for $15 \mathrm{~min}$ at $5000 \times \mathrm{g}$.

According to the manufacturer's instructions, the TRIzol ${ }^{\circledR}$ reagent (Carlsbad, CA, USA) was used to extract from the destructive cylindrical Rocapone tissue. Total RNA was isolated and genomic DNA was removed by DNA enzyme I (TaKaRa, Dalian, China). The integrity and purity of the total RNA were determined by a 2100 Biological Analyzer (Santa Clara Agilent Technologies, USA) and quantified using ND-2000 (Deweymington NanoDrop Thermo Scientific, USA). Only the high-quality RNA samples $(\mathrm{OD} 260 / 280=1.8-2.2, \mathrm{OD} 260 / 230 \geq 2.0, \mathrm{RIN} \geq 8.0,28 \mathrm{~s}: 18 \mathrm{~s} \geq 1.0,>2 \mu \mathrm{g})$ were utilized for constructing the sequencing library.

2.3. Library preparation and Illumina Novaseq 6000 Sequencing

According to the manufacturer's specification (Illumina, San Diego, CA, USA), RNA purification, reverse transcription, library construction, and sequencing were carried out in Shanghai Mayobio Biopharmaceutical Biotechnology Co., Ltd. (Shanghai, China). The destructive Illumina TruseqTM RNA 
transcripts group library was prepared by using the I. robusta RNAseq sample preparation kit (San Diego, CA, USA). Total RNA was separated by oligosaccharide adsorption beads and denatured by pyrolysis buffer. These short segments were used as templates, and double-stranded cDNA was synthesized by superscript double-stranded cDNA synthesis kit (Invitrogen, CA, USA) and random hexagonal primers (Illumina). Then, according to the library construction scheme of Illumina, the synthesized cDNA was repaired, phosphorylated, and a-base addition was carried out. A library of cDNA target fragments of 200-300 bp was selected on $2 \%$ low range hyperagarose and amplified by 15 cycles of PCR using Phusion DNA polymerase (New England Biolabs, Boston, MA, USA). After TBS380 quantification, two RNAseq libraries were read by $2 \times 150$ bp pairs on Illumina Novaseq 6000 sequencer (Illumina).

\subsection{De novo assembly and annotation}

End readings assembled from scratch and aligned in pairs are trimmed and quality controlled by Seqprep (https://github.com/jstjohn/seqprep) and Sickle (https://github.com/najoshi/sickel) using default parameters. Then, the trinity was assembled from scratch (http://trinityrnaseq.sourceforge.net/)using clean data from the sample (xx) [25]. All assembled transcripts were searched based on the NCBI protein non-reductant (NR), and the KEGG database uses Blastx to identify proteins with the highest sequence similarity to a given transcript to retrieve the functional annotations. A typical cutoff value was set at < $1.0 \times 10-5$. The blast2go (http://www.blast2go.com/b2ghome) program was used to obtain unique assembly transcriptsgo annotations that describe the biological processes, molecular functions, and cellular components[26]. Metabolic pathway was analyzed using KEGG (http://www.genome.jp/KEGG/) [27].

\subsection{Differential expression analysis and functional enrichment}

Differential expression analysis and functional enrichment identification of differentially expressed gene (DEGs) in two different samples were used to calculate the expression level of each transcript according to the exon per thousand base fragment/million mapped reads (frkm) method. Rsem (http://deweylab.biostat.wisc.edu/rsem/) was used to quantify the abundance of genes and subtypes[28]. The empirical analysis of digital gene expression was performed in $\mathrm{R}$ statistical software package Edger (R, http://www.bioconductor.org/packages/2.12/bioc/html/edger.html) to assess the differential expression[29]. In addition, functional enrichment analysis, including GO and KEGG, was carried out to identify the DEGs that was significantly enriched in GO terms and metabolic pathways when Bonferroni corrected P-value $\leq$ 0.05. Goatools (https://github.com/tanghaibao/goatools) and Kobas (http://kobas.cbi.pku.edu.cn/home.do) were used for GO functional enrichment and KEGG pathway analysis[30].

\subsection{Quantitative RT-PCR validation of target genes}

Each RNA sample was adjusted to $1 \mu \mathrm{g} / \mu \mathrm{L}$ with nuclease-free water. An equivalent of $2 \mu \mathrm{g}$ of total RNA was reverse transcribed in a $20 \mu \mathrm{L}$ reaction volume using the Prime ScriptTM RT reagent Kit with gDNA Eraser (TaKaRa). The I. robusta $18 \mathrm{~S}$ gene was used as an internal control. qRT-PCR was performed using 
the SYBR ${ }^{\circledR}$ Premix Ex Taq ${ }^{\text {TM }}$ (Tli-RNase H Plus) (TaKaRa), according to the manufacturer's protocol. A no template control (NTC) (nuclease-free water) was included to detect the contamination and determine the degree of dimer formation. The qRT-PCR analysis was performed in triplicate for each extracted RNA sample. PCR amplifications were performed using a Roche Light Cycler 480 (Indianapolis, IN, USA) in a $20 \mu \mathrm{L}$ reaction volume. The data were analyzed using the Light Cycler ${ }^{\circledR} 480$ software. A relative quantitative method $(\Delta \Delta \mathrm{Ct})$ was used to evaluate the quantitative variation 60 . The sequences of the primer sets are listed in Table S4.

\section{Declarations}

Declarations

Ethics approval and consent to participate

Not applicable

Consent for publication

Not applicable

Availability of data and material

The datasets generated and/or analysed during the current study are available in the NCBI repository, under the Bioproject accession number PRJNA575915.

\section{References}

1. Wu L, Zhao Y, Guan Y, Pang S. A review on studies of the reason and control methods of succession cropping obstacle of Panax ginseng CA Mey. Special Wild Economic Animal Plant Research 2008;2:68-72.

2. Li Y, Huang $X$, Ding W. Autotoxicity of Panax ginseng rhizosphere and non-rhizosphere soil extracts on early seedlings growth and identification of chemicals. Allelopathy Journal 2011;28.

3. Nicol RW, Yousef L, Traquair JA, Bernards MA. Ginsenosides stimulate the growth of soilborne pathogens of American ginseng. Phytochemistry 2003;64:257-264.

4. Mukerji KG. Allelochemicals: biological control of plant pathogens and diseases: Springer, 2006.

5. Zhao X, Gao J, Song C, et al. Fungal sensitivity to and enzymatic deglycosylation of ginsenosides. Phytochemistry 2012;78:65-71.

6. Gul S, Whalen JK, Thomas BW, Sachdeva V, Deng H. Physico-chemical properties and microbial responses in biochar-amended soils: mechanisms and future directions. Agriculture, Ecosystems \& Environment 2015;206:46-59. 
7. Farh ME-A, Kim Y-J, Kim Y-J, Yang D-C. Cylindrocarpon destructans/llyonectria radicicola-species complex: Causative agent of ginseng root-rot disease and rusty symptoms. Journal of ginseng research 2018;42:9-15.

8. Klosterman SJ, Subbarao KV, Kang S, et al. Comparative genomics yields insights into niche adaptation of plant vascular wilt pathogens. PLoS pathogens 2011;7:e1002137.

9. Foyer $\mathrm{CH}$, Noctor $\mathrm{G}$, Hodges $\mathrm{M}$. Respiration and nitrogen assimilation: targeting mitochondriaassociated metabolism as a means to enhance nitrogen use efficiency. Journal of Experimental Botany 2011;62:1467-1482.

10. Winnenburg R, Urban M, Beacham A, et al. PHI-base update: additions to the pathogen-host interaction database. Nucleic acids research 2007;36:D572-D576.

11. Baldwin TK, Winnenburg R, Urban M, Rawlings C, Koehler J, Hammond-Kosack KE. The pathogenhost interactions database (PHI-base) provides insights into generic and novel themes of pathogenicity. Molecular plant-microbe interactions 2006;19:1451-1462.

12. Tournas V, Kohn J, Katsoudas E. Interactions between various microbes and ginseng botanicals. Critical reviews in microbiology 2011;37:113-120.

13. Shinzato C, Shoguchi E, Kawashima T, et al. Using the Acropora digitifera genome to understand coral responses to environmental change. Nature 2011;476:320.

14. Abro MA, Lecompte F, Bryone F, Nicot PC. Nitrogen fertilization of the host plant influences production and pathogenicity of Botrytis cinerea secondary inoculum. Phytopathology 2013;103:261-267.

15. Marzluf GA. Genetic regulation of nitrogen metabolism in the fungi. Microbiol Mol Biol Rev 1997;61:17-32.

16. Usaite R, Patil KR, Grotkjær T, Nielsen J, Regenberg B. Global transcriptional and physiological responses of Saccharomyces cerevisiae to ammonium, L-alanine, or L-glutamine limitation. Appl Environ Microbiol 2006;72:6194-6203.

17. Marini A-M, Soussi-Boudekou S, Vissers S, Andre B. A family of ammonium transporters in Saccharomyces cerevisiae. Molecular and cellular biology 1997;17:4282-4293.

18. Zeng X, Zhang Q, Kudinha T, Kong F. Comparative genome and transcriptome study of the gene expression difference between pathogenic and environmental strains of Prototheca zopfii. Frontiers in microbiology 2019;10:443.

19. Zhang Z, Wang J, Chai R, et al. An S-(hydroxymethyl) glutathione dehydrogenase is involved in conidiation and full virulence in the rice blast fungus Magnaporthe oryzae. PLoS One 2015;10:e0120627.

20. Liu W, Yu Y-H, Cao S-Y, et al. Transcriptome profiling of Xanthomonas campestris pv. campestris grown in minimal medium MMX and rich medium NYG. Research in microbiology 2013;164:466-479.

21. Corral-Ramos C, Roncero MIG. Glycogen catabolism, but not its biosynthesis, affects virulence of Fusarium oxysporum on the plant host. Fungal Genetics and Biology 2015;77:40-49. 
22. Urban M, Mott E, Farley T, Hammond-Kosack K. The Fusarium graminearum MAP1 gene is essential for pathogenicity and development of perithecia. Molecular plant pathology 2003;4:347-359.

23. Lin Z, Wei J, Zhang M, et al. Identification and characterization of a new fungal pathogen causing twisted leaf disease of sugarcane in China. Plant disease 2015;99:325-332.

24. Bhatnagar D, Ehrlich KC, Cleveland TE. Oxidation-reduction reactions in biosynthesis of secondary metabolites. Handbook of applied mycology 1991;5:255-286.

25. Grabherr MG, Haas BJ, Yassour M, et al. Full-length transcriptome assembly from RNA-Seq data without a reference genome. Nature biotechnology 2011;29:644.

26. Conesa A, Götz S, García-Gómez JM, Terol J, Talón M, Robles M. Blast2GO: a universal tool for annotation, visualization and analysis in functional genomics research. Bioinformatics 2005;21:3674-3676.

27. Kanehisa M, Furumichi M, Tanabe M, Sato Y, Morishima K. KEGG: new perspectives on genomes, pathways, diseases and drugs. Nucleic acids research 2016;45:D353-D361.

28. Li B, Dewey CN. RSEM: accurate transcript quantification from RNA-Seq data with or without a reference genome. BMC bioinformatics 2011;12:323.

29. Robinson MD, McCarthy DJ, Smyth GK. edgeR: a Bioconductor package for differential expression analysis of digital gene expression data. Bioinformatics 2010;26:139-140.

30. Xie C, Mao X, Huang J, et al. KOBAS 2.0: a web server for annotation and identification of enriched pathways and diseases. Nucleic acids research 2011;39:W316-W322.

\section{Figures}


A
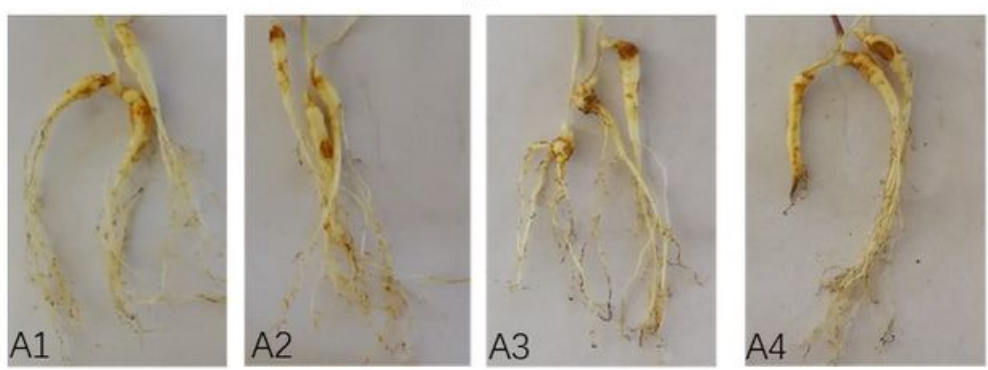

B

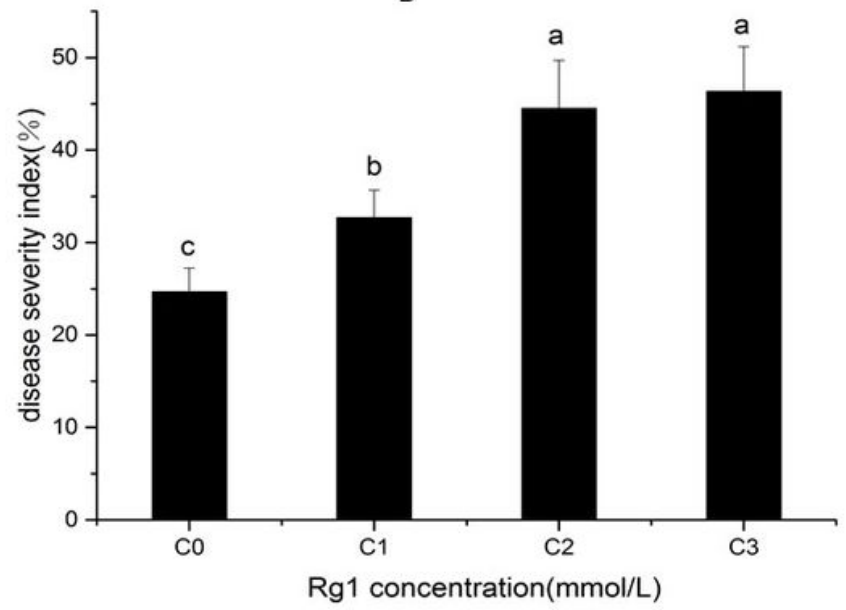

\section{Figure 1}

The disease symptom (A) and disease severity index (B) of ginseng plants treated with different concentrations of ginsenoside Rg1: $0.5 \mathrm{mM}$ (A2), 1 mM (A3), $2 \mathrm{mM}$ (A4), and no Rg1 control (A1). (A) Images acquired on day 30 after inoculation. The average severity index of the cylindrical root rot of (B) ginseng (\%). 

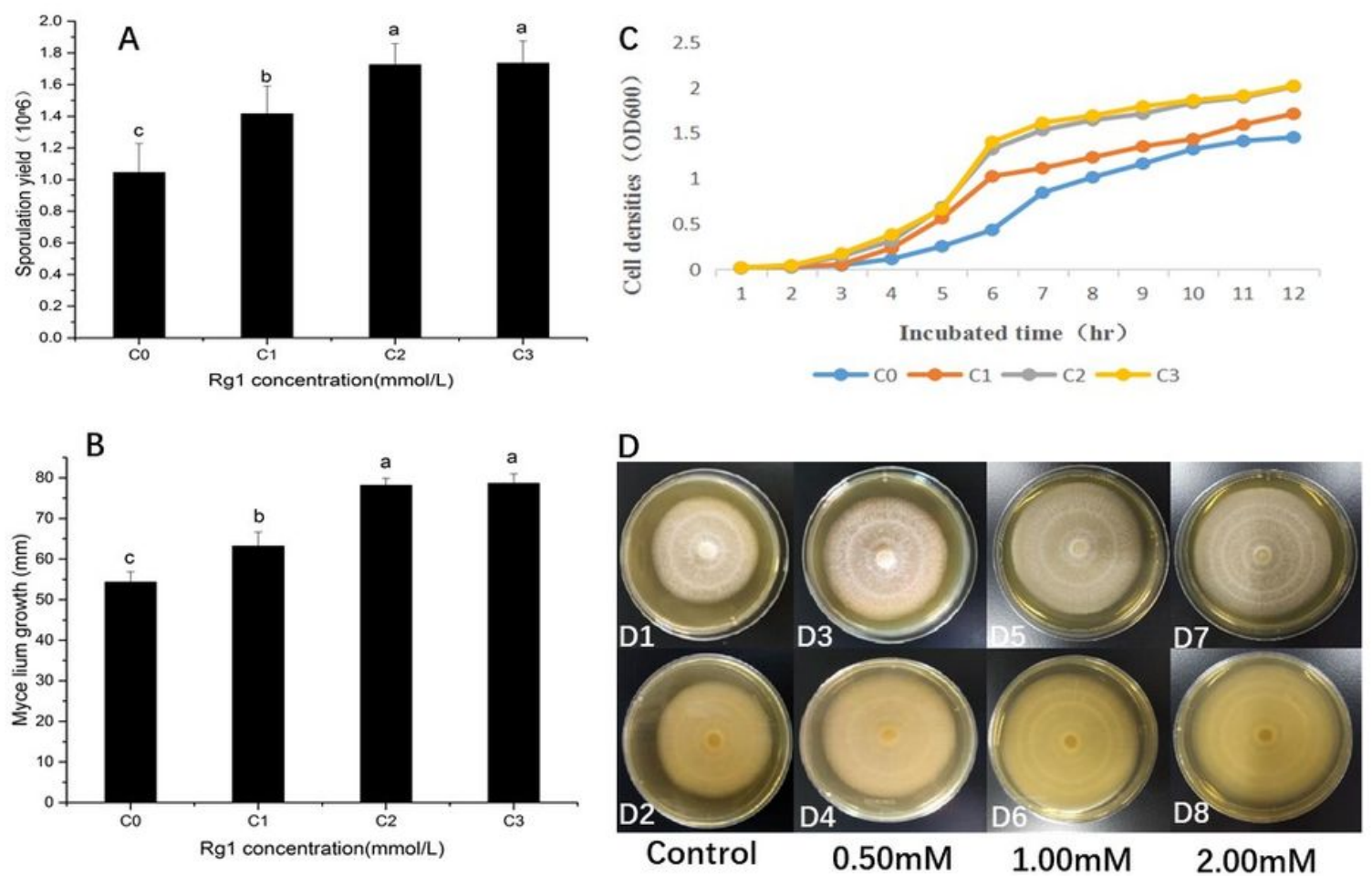

\section{Figure 2}

Effects of ginsenoside Rg1 on the growth of I. robusta cultured in vitro. (A) Sporulation production at DAI 3 (3 days after inoculation); (B) Fungal cell densities (OD600) at logistic increment; (C) Fungal mycelial growth at increment; (D) Colonies of I. robusta observed at DAI 3 in no ginsenoside Rg1 control (D1, top view; D2, bottom view), $0.5 \mathrm{mM}$ ginsenoside Rg1 (D3, top view; D4, bottom view), $1 \mathrm{mM}$ ginsenoside Rg1 (D5, top view; D6, bottom view), and 2mM ginsenoside Rg1 (D7, top view; D8, bottom view). The different letters in Fig. 2A and Fig. 2B indicate a highly significant difference $(P<0.01)$. The error bar represents the standard deviation of at least three repeats.
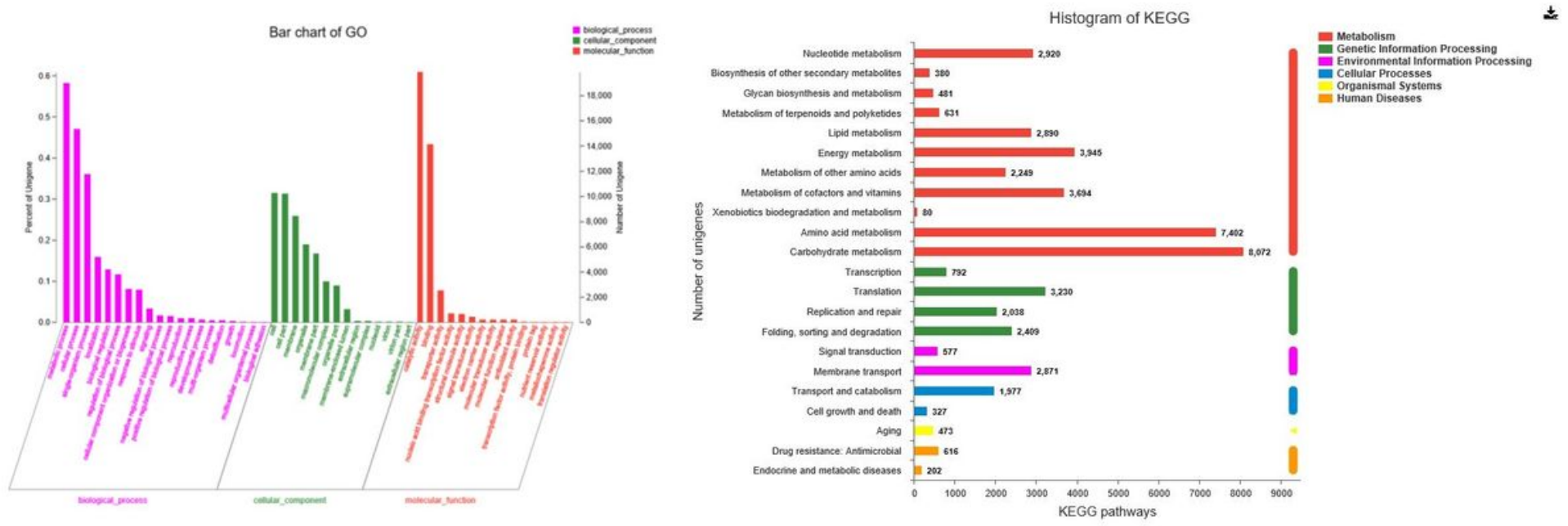

Figure 3 
Transcriptome functional annotation analysis. (A) GO classification of the unigenes. Based on the functional annotation, these unigenes were grouped into three categories: biological process, cellular component, and molecular function. (B) KEGG classified these metabolic pathways into seven categories: metabolism, genetic information processing, environmental information processing, cellular processes, organismal systems, human diseases, drug development).

GO enrichment analysis(CK_Third_vs_G_Third_T)

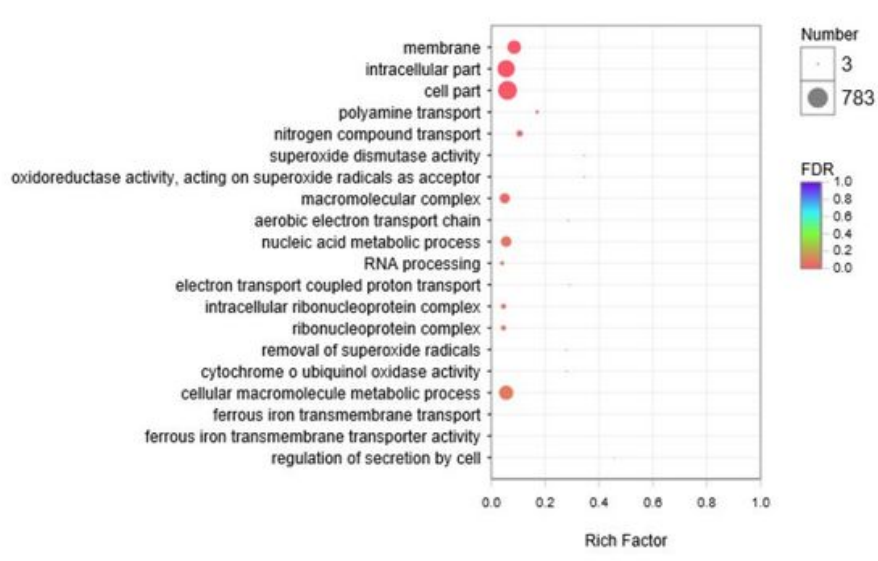

KEGG enrichment analysis(CK_Third_vs_G_Third_G)

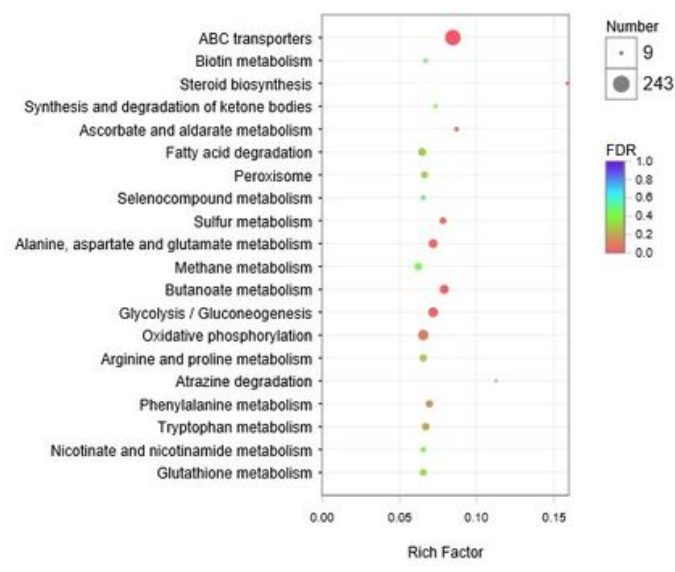

\section{Figure 4}

Differential gene enrichment analysis. (A) GO enrichment analysis and (B) KEGG enrichment analysis. The ratio of the number of unigenes commented to the GO/KEGG term in the gene set to the number of unigenes commented to the GO/KEGG term. The larger the ratio, the greater the degree of enrichment. The size of the point represents the number of unigenes in the GO/KEGG term, and the color of the point corresponds to a different FDR (pvaule_corrected) range. 


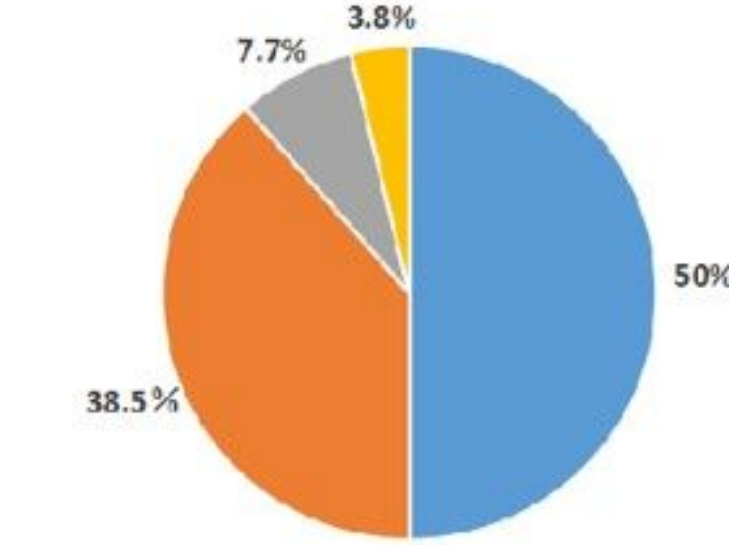

| unaffeeted_pathogenicity = virulenoe || lethal = hypervirulenec

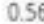

0.54

0.52

0.5

0.48

0.46

0.44

0.42

Up

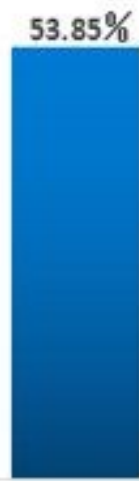

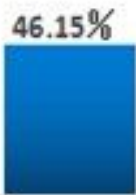

Down

A

B

Figure 5

(A) DEGs related to Phi and the phenotype of pathogen. (B)Bar graph presents the expression estimates for genes differentially regulated according to the $\mathrm{Rg} 1$ treatment.

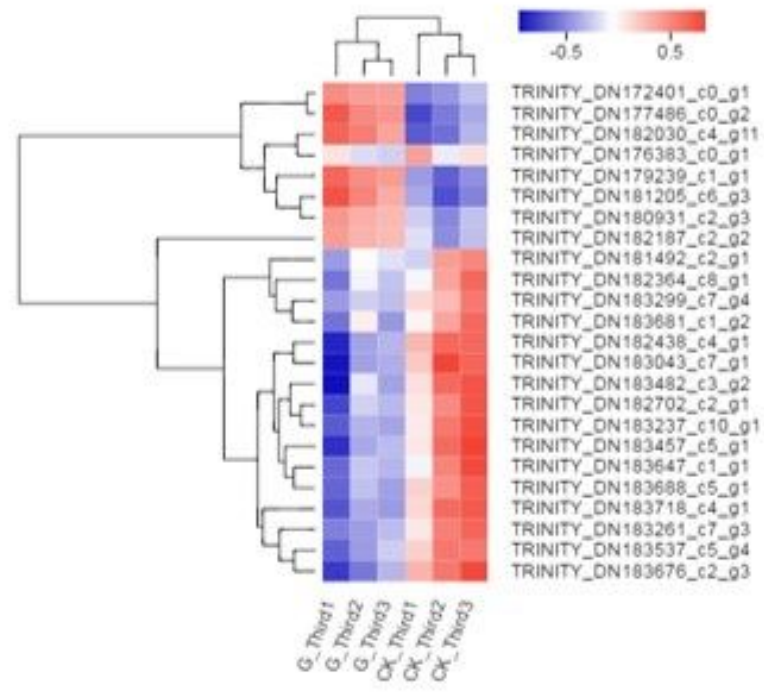

A

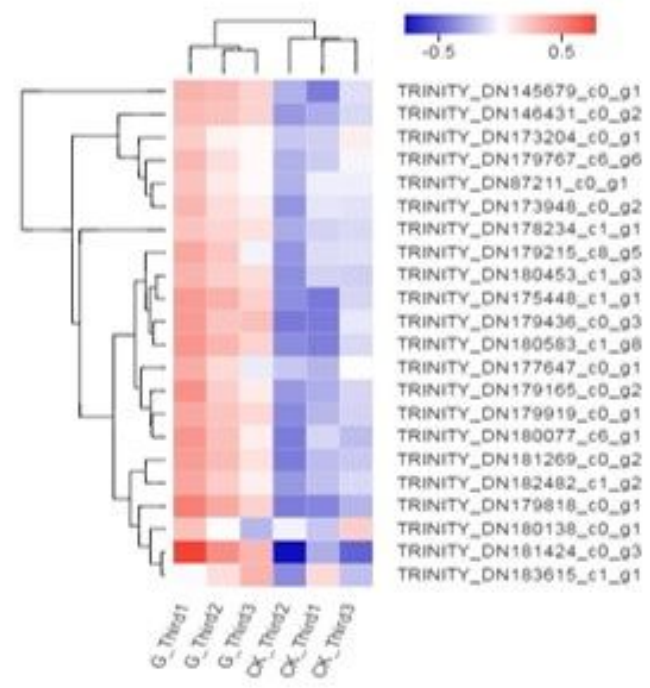

B

Figure 6 
(A) Cluster analysis of gene expression patterns selected in Phi. (B) Cluster analysis of cell growth-related gene expression patterns.

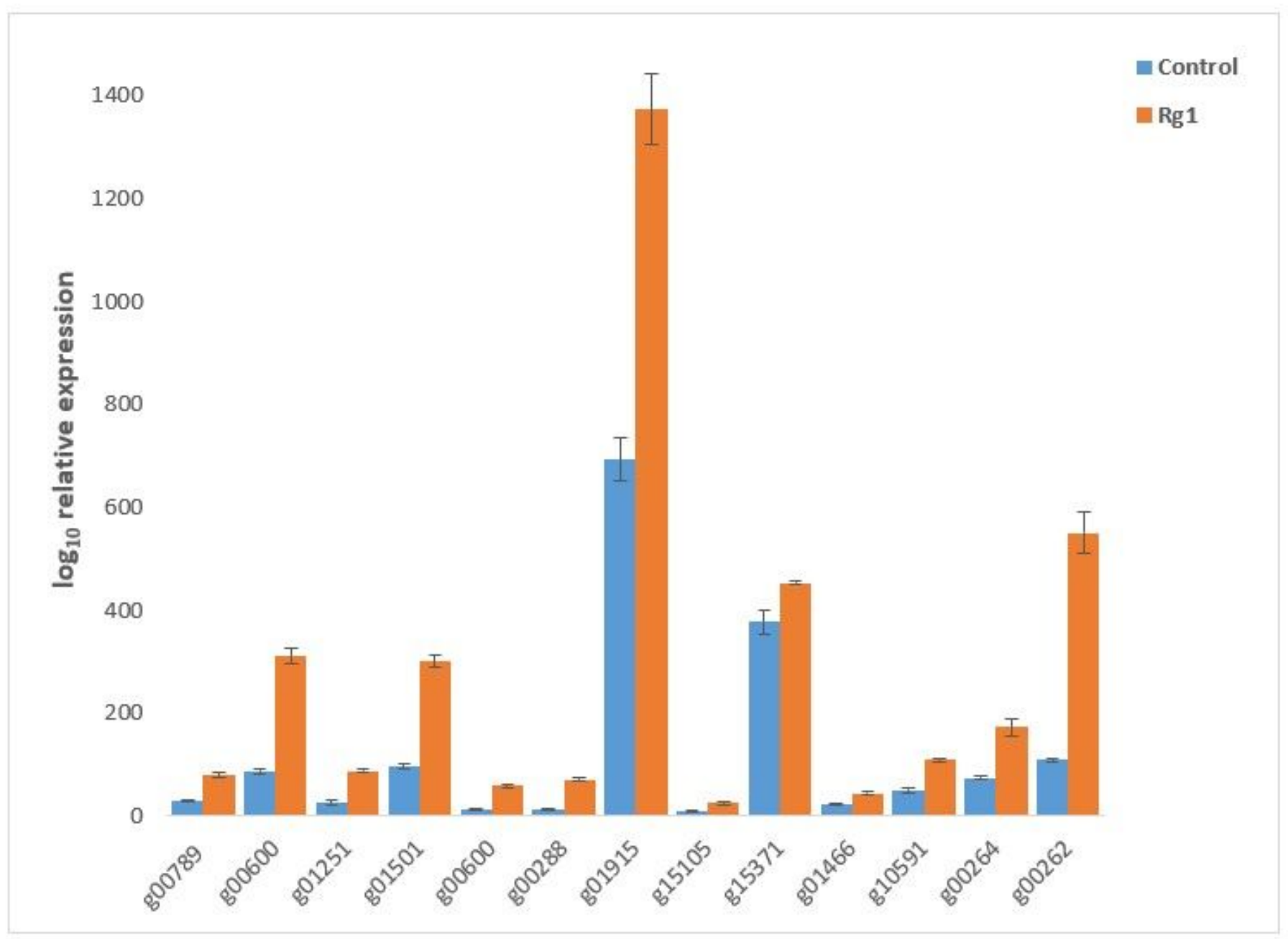

Figure 7

qRT-PCR analysis of selected genes. 